\title{
Safety profile of brentuximab vedotin in Japanese patients with relapsed/refractory Hodgkin lymphoma or systemic anaplastic large cell lymphoma: a post-marketing surveillance study
}

\author{
Koji Izutsu $^{1,2} \circledast$ - Michinori Ogura ${ }^{3} \cdot$ Kensei Tobinai $^{1} \cdot$ Kiyohiko Hatake $^{4,5} \cdot$ Shigeru Sakamoto $^{6} \cdot$ Masanori Nishimura $^{7}$. \\ Miyako Hoshino ${ }^{5}$
}

Received: 15 April 2020 / Revised: 4 November 2020 / Accepted: 6 November 2020 / Published online: 3 January 2021

(c) Japanese Society of Hematology 2021

\begin{abstract}
Brentuximab vedotin (BV) was initially approved in Japan for the treatment of relapsed/refractory (R/R) CD30-positive Hodgkin lymphoma (HL) and systemic anaplastic large cell lymphoma (sALCL). As requested by the Japanese Ministry of Health, Labour and Welfare, we conducted a post-marketing surveillance (PMS) study to assess the safety of BV in Japanese patients with R/R HL or sALCL. PMS forms were collected from 284 patients (182 with HL, 101 with sALCL and one with another lymphoma) treated between April and September 2014. The median age was 62 (range 14-93) years and the median number of treatment cycles was 5.5 for HL and 4 for sALCL. Adverse drug reactions (ADRs) were reported in $74.3 \%$ of patients. The most commonly observed ADRs included peripheral sensory neuropathy (39.1\%; grade $\geq 3,6.3 \%$ ), neutropenia (34.5\%; grade $\geq 3,22.2 \%$ ) and lymphopenia ( $7.0 \%$; grade $\geq 3,5.3 \%)$. Ten patients had fatal ADRs including interstitial lung disease $(n=3)$. This study showed that BV has an acceptable safety profile in Japanese patients with $\mathrm{R} / \mathrm{R} \mathrm{HL}$ and $\mathrm{R} / \mathrm{R}$ sALCL in the clinical practice setting. However, close monitoring rare, but potentially fatal, ADRs such as pulmonary toxicity may be warranted, especially in patients with prior or ongoing pulmonary disorders.
\end{abstract}

Keywords Brentuximab vedotin $\cdot$ Post-marketing surveillance $\cdot$ Hodgkin lymphoma $\cdot$ Systemic anaplastic large cell lymphoma $\cdot$ Safety profile

Electronic supplementary material The online version of this article (https://doi.org/10.1007/s12185-020-03039-w) contains supplementary material, which is available to authorized users.

Miyako Hoshino

miyako.hoshino@takeda.com

1 Department of Hematology, National Cancer Center Hospital, Tokyo, Japan

2 Department of Hematology, Toranomon Hospital, Tokyo, Japan

3 Department of Hematology/Oncology, Kasugai Municipal Hospital, Kasugai, Japan

4 Department of Hematology, International University of Health and Welfare (IUHW) School of Medicine, Tokyo, Japan

\section{Introduction}

Brentuximab vedotin is an antibody-drug conjugate comprising a CD30-directed antibody linked to a microtubuledisrupting agent, monomethyl auristatin E [1]. Brentuximab vedotin was initially approved in the USA in 2011 [2] based on the results from two pivotal phase 2 studies

5 Department of Hematology and Oncology, The Cancer Institute Hospital of Japanese Foundation for Cancer Research, Tokyo, Japan

6 Japan Medical Office, Japan Pharma Business Unit, Takeda Pharmaceutical Co. Ltd., 1-1, Nihonbashi-Honcho 2-chome, Chuo-ku, Tokyo 103-8668, Japan

7 Pharmacovigilance, Takeda Development Center Japan, Takeda Pharmaceutical Co. Ltd., Osaka, Japan 
in relapsed/refractory (R/R) Hodgkin lymphoma (HL) [3] and systemic anaplastic large cell lymphoma (sALCL) [4]. In both studies, brentuximab vedotin was associated with high response rates and promising progression-free survival (PFS) and overall survival (OS), along with a number of important safety signals including peripheral neuropathy, infections, neutropenia, and infusion reactions [3-6]. Additionally, acute pulmonary toxicity is a rare adverse event (AE) associated with brentuximab vedotin use that is potentially life-threatening [7].

Brentuximab vedotin was approved in Japan in 2014 for patients with R/R CD30-positive HL or sALCL based on a Japanese phase $1 / 2$ study to evaluate the safety, pharmacokinetics, and efficacy of brentuximab vedotin in Japanese patients with R/R HL $(n=14)$ or R/R sALCL $(n=6)$ [8]. This study demonstrated that response rates and reported AEs were similar to those observed in the previous international studies $[3,4,8]$.

Nevertheless, further investigation was required by the Ministry of Health, Labour and Welfare in Japan as the number of patients enrolled in the Japanese study above was limited. Therefore, we conducted a post-marketing surveillance study to evaluate the safety profile of brentuximab vedotin in clinical practice in Japan. In addition, we collected exploratory data on antitumor activity and OS in this surveillance.

\section{Patients and methods}

\section{Study design}

This nation wide non-interventional observational study included all patients who were treated with brentuximab vedotin in Japan between April 17 and September 30, 2014 , according to a ministerial ordinance concerning standards for implementing post-marketing surveillance studies of drugs (Good Post-marketing Study Practices [GPSP]) in Japan. Case report forms were scheduled to be collected from the investigating physicians within a month following the 16th cycle or treatment discontinuation, whichever occurred first. The aim of this surveillance study was to collect safety data of brentuximab vedotin in clinical practice in Japan; collection of other data was not strictly enforced and duration of follow-up for AEs was not strictly defined. Approvals from each institutional ethics committee and written informed consent from patients were not mandatory in this study, because GPSP does not require them. The study protocol was registered with Japan Pharmaceutical Information Center-Clinical Trials Information (JapicCTI-142455) and ClinicalTrials.gov (NCT02139592).

\section{Treatment}

Brentuximab vedotin was administered in accordance with the Japanese package insert [9], in which the recommended starting dose is $1.8 \mathrm{mg} / \mathrm{kg}$ every 3 weeks.

\section{Assessment}

Patients were observed from the start of brentuximab vedotin treatment through 16 cycles or until treatment discontinuation. The follow-up period of up to 16 cycles was defined based on a previous clinical study investigating the safety of brentuximab vedotin beyond 16 cycles (NCT00947856). The following details were recorded regarding each patient's treatment: use of premedication for infusion reactions, receipt of hematopoietic stem cell transplantation (HSCT) after brentuximab vedotin treatment, antitumor treatment for HL or SALCL other than brentuximab vedotin and use of prophylactic agents against infection.

Throughout the observation period, AEs were monitored and recorded by the investigating physicians until the final assessment. After the final assessment, recorded AEs were coded using the Medical Dictionary for Regulatory Activities Japanese language version (MedDRA/J, version 19.1) and graded according to the Common Terminology Criteria for Adverse Events (CTCAE, version 4.0). Progression of the primary disease was not considered as an AE, unless the disease worsened abnormally and was described by the investigating physician as an AE. AEs for which a causal relationship to brentuximab vedotin was at least a reasonable possibility (i.e., the relationship could not be ruled out) as deemed by the investigating physician were defined as adverse drug reactions (ADRs). ADRs of special interest (ADRSIs) for this study were peripheral neuropathy (including sensory and motor), infections, neutropenia, infusion reactions, and pulmonary toxicity. The definitions of each of the ADRSIs were established using System Organ Class or Preferred Terms within MedDRA/J.

Patients were assessed for antitumor activity during treatment per routine clinical practice at the study sites, including the use of positron emission tomography (PET). Using these assessments, best response was determined according to the Revised Response Criteria for Malignant Lymphoma [10]. Survival status was confirmed for each patient at the time of their final observation date; for patients who died, the date of death was recorded.

\section{Statistical analysis}

To observe evaluable occurrences of major events observed in the pivotal phase 2 studies with a frequency of $\geq 10 \%$, 
the sample size was planned to be 140 patients $[3,4]$. All patients whose surveillance form was provided were included in the safety analysis. Patients treated for R/R HL or sALCL were included in the effectiveness analysis. All data were tabulated using descriptive statistics. OS was assessed using Kaplan-Meier methodology. OS was defined as the time from a patient's first dose of brentuximab vedotin to their final observation date. Statistical analysis was conducted using SAS version 9.2 (for the final analysis set) and SAS version 9.4 (for additional analyses).

\section{Results}

\section{Patient demographics and baseline characteristics}

A total of 292 patients from 198 institutions were registered for this study, and completed surveillance forms were collected from 284 patients, including 182 with HL, 101 with SALCL, and one with diffuse large B-cell lymphoma (DLBCL) (Fig. 1). All 284 patients were included in the safety analysis. The patient with DLBCL and three patients with HL or SALCL who received brentuximab vedotin as first-line therapy were excluded from the effectiveness analysis (Fig. 1).

Patient demographics and baseline disease characteristics are shown in Table 1. The median age (range) of the total, HL and sALCL populations was 62.0 (14-93), 60.5 (16-91) and 64.0 (14-93) years, respectively. Of the total patient population, $40.1 \%, 36.6 \%$, and $23.2 \%$ of patients had an Eastern Cooperative Oncology Group (ECOG) performance status of 0,1 or $\geq 2$, respectively. Stage IV disease was observed in $46.7 \%$ and $54.5 \%$ of patients with HL and sALCL, respectively. Of the total patient population, $10.2 \%$ had a history of or ongoing pulmonary disorders. Patients had received a median of 2.0 (range 1-13) prior treatment regimens, and $22.5 \%$ of patients had received prior autologous and/or allogenic HSCT. In patients with sALCL, 76.2\% were negative for anaplastic lymphoma kinase (ALK) and 8.9\% had unknown ALK status.

\section{Treatment}

The extent of exposure of patients to brentuximab vedotin is summarized in Table 2. In total, $87.4 \%$ of patients with HL and $86.1 \%$ of those with sALCL received a starting dose of $1.8 \mathrm{mg} / \mathrm{kg}$ and $77.5 \%$ of patients with HL and $71.3 \%$ of those with sALCL received a mean dose per cycle of $1.8 \mathrm{mg} /$ $\mathrm{kg}$ throughout treatment. The median number of delivered treatment cycles in HL and sALCL were 5.5 (range 1-16) and 4 (range 1-17), respectively. The proportion of patients who received the full 16 cycles of brentuximab vedotin treatment was $11.0 \%$ in HL and $10.9 \%$ in sALCL; one patient with sALCL received 17 cycles of treatment. Reasons for treatment discontinuation were lack of efficacy in $40.7 \%$ and $39.6 \%$, AEs in $23.1 \%$ and $20.8 \%$, and achievement of treatment objectives according to the physician's decision in $19.2 \%$ and $24.8 \%$ of patients with HL and sALCL, respectively (Table 2).

Prior to the first dose of brentuximab vedotin, premedication for infusion reactions was administered in $59.9 \%$ and $74.3 \%$ of patients in the HL and sALCL, respectively. Corticosteroids, as premedication, were given to approximately $60 \%$ of patients.

In the HL and sALCL, $9.9 \%$ and $12.9 \%$ of patients, respectively, received combination antitumor therapies with brentuximab vedotin for treatment of their disease. The most common drugs were prednisolone and dexamethasone,
Fig. 1 Composition of registered patients and analysis sets

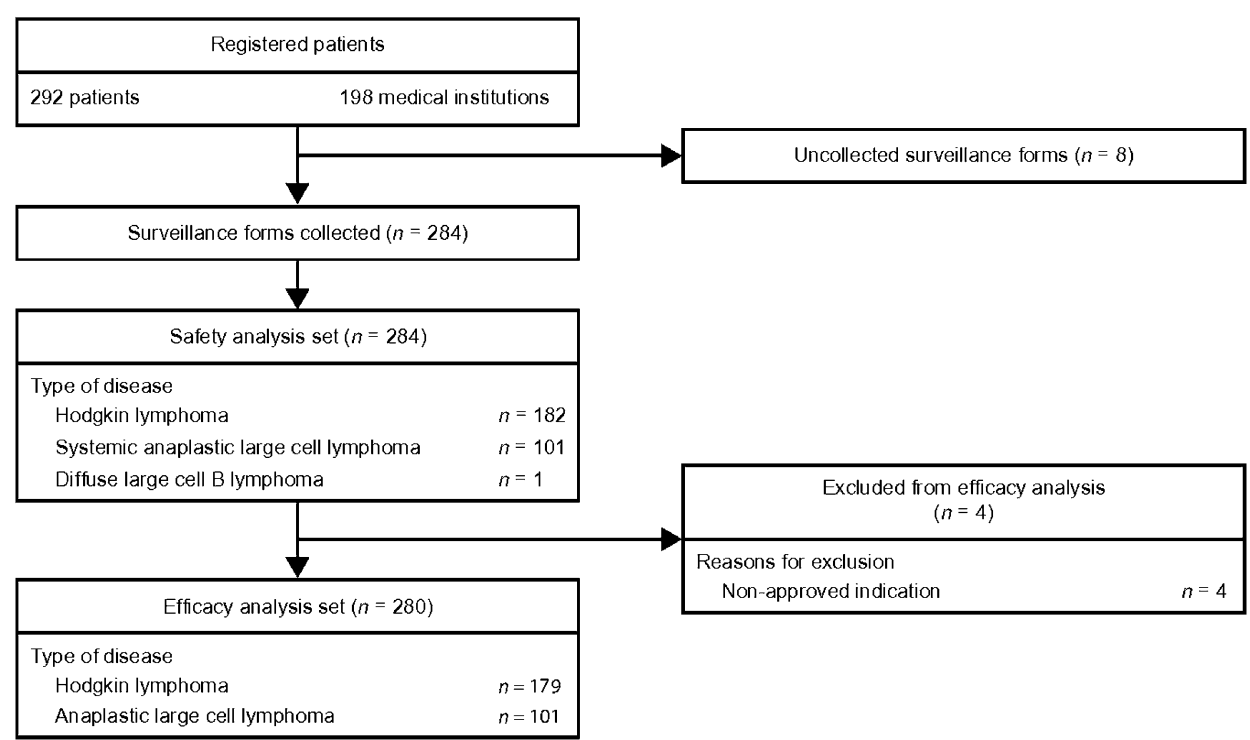


Table 1 Baseline characteristics of patients

\begin{tabular}{|c|c|c|c|}
\hline & $\begin{array}{l}\text { Total } \\
(N=284)^{\mathrm{a}}\end{array}$ & $\begin{array}{l}\text { HL } \\
(n=182)\end{array}$ & $\begin{array}{l}\text { sALCL } \\
(n=101)\end{array}$ \\
\hline Male & $184(64.8)$ & $115(63.2)$ & $69(68.3)$ \\
\hline $\begin{array}{l}\text { Age, years, median } \\
\text { (range) }\end{array}$ & $62.0(14-93)$ & $60.5(16-91)$ & $64.0(14-93)$ \\
\hline \multicolumn{4}{|c|}{ Ann Arbor disease stage at start of BV treatment } \\
\hline I & $10(3.5)$ & $5(2.7)$ & $5(5.0)$ \\
\hline II & $63(22.2)$ & $45(24.7)$ & $18(17.8)$ \\
\hline III & $70(24.6)$ & $47(25.8)$ & $22(21.8)$ \\
\hline IV & $140(49.3)$ & $85(46.7)$ & $55(54.5)$ \\
\hline Unknown & $1(0.4)$ & 0 & $1(1.0)$ \\
\hline \multicolumn{4}{|c|}{ ECOG performance status } \\
\hline 0 & $114(40.1)$ & $79(43.4)$ & $35(34.7)$ \\
\hline 1 & $104(36.6)$ & $67(36.8)$ & $37(36.6)$ \\
\hline 2 & $32(11.3)$ & $19(10.4)$ & $13(12.9)$ \\
\hline 3 & $19(6.7)$ & $7(3.8)$ & $11(10.9)$ \\
\hline 4 & $15(5.3)$ & $10(5.5)$ & $5(5.0)$ \\
\hline $\begin{array}{l}\text { History of pulmonary } \\
\text { disorders }\end{array}$ & $9(3.2)$ & $9(4.9)$ & 0 \\
\hline $\begin{array}{l}\text { Ongoing pulmonary } \\
\text { disorders }\end{array}$ & $20(7.0)$ & $16(8.8)$ & $4(4.0)$ \\
\hline \multicolumn{4}{|c|}{ Ongoing peripheral neuropathy } \\
\hline None & $230(81.0)$ & $147(80.8)$ & $82(81.2)$ \\
\hline Grade 1 & $35(12.3)$ & $26(14.3)$ & $9(8.9)$ \\
\hline Grade 2 & $17(6.0)$ & $8(4.4)$ & $9(8.9)$ \\
\hline Grade 3 & $1(0.4)$ & $1(0.5)$ & 0 \\
\hline Grade 4 & 0 & 0 & 0 \\
\hline Grade unknown & $1(0.4)$ & 0 & $1(1.0)$ \\
\hline $\begin{array}{l}\text { Received prior chemo- } \\
\text { therapy }\end{array}$ & $279(98.2)$ & $178(97.8)$ & $100(99.0)$ \\
\hline ABVD & $165(58.1)$ & $163(89.6)$ & $2(2.0)$ \\
\hline $\mathrm{CHOP}$ & $88(31.0)$ & $19(10.4)$ & $69(68.3)$ \\
\hline Others & $215(75.7)$ & $134(73.6)$ & $80(79.2)$ \\
\hline \multicolumn{4}{|c|}{ Number of prior drug treatment regimens } \\
\hline & $n=279$ & $n=178$ & $n=100$ \\
\hline Median (range) & $2.0(1-13)$ & $2.0(1-9)$ & $2.0(1-13)$ \\
\hline $\begin{array}{l}\text { Received prior radio- } \\
\text { therapy }\end{array}$ & $105(37.0)$ & $74(40.7)$ & $31(30.7)$ \\
\hline \multicolumn{4}{|l|}{ Prior HSCT } \\
\hline Autologous & $43(15.1)$ & $33(18.1)$ & $10(9.9)$ \\
\hline Allogeneic & $9(3.2)$ & $7(3.8)$ & $2(2.0)$ \\
\hline $\begin{array}{l}\text { Autologous and allo- } \\
\text { geneic }\end{array}$ & $12(4.2)$ & $8(4.4)$ & $4(4.0)$ \\
\hline
\end{tabular}

${ }^{a}$ One patient had diffuse large B-cell lymphoma

Number of patients (\%) are shown unless indicated otherwise

$A B V D$ doxorubicin, bleomycin, vinblastine and dacarbazine, $B V$ brentuximab vedotin, $C H O P$ cyclophosphamide, doxorubicin, vincristine and prednisolone, ECOG Eastern Cooperative Oncology Group, $H L$ Hodgkin lymphoma, HSCT hematopoietic stem cell transplant, sALCL, systemic anaplastic large cell lymphoma which were given to 16 (5.6\%) patients with either disease; a total of $15(5.3 \%)$ patients received one or more cytotoxic agents (Supplementary Table 1).

\section{Safety}

In the safety population, the incidence of ADRs was $74.3 \%$, with the most commonly reported ADRs being peripheral sensory neuropathy $(39.1 \%$; grade $\geq 3,6.3 \%)$, neutropenia $(34.5 \%$; grade $\geq 3,22.2 \%)$, and lymphopenia $(7.0 \%$; grade $\geq 3,5.3 \%$ ) (Table 3 ).

The most common serious ADRs that occurred in $\geq 2 \%$ of all patients were neutropenia (4.9\%), interstitial lung disease (3.9\%), pneumonia (2.1\%), and peripheral sensory neuropathy (2.1\%) (Table 4). Ten patients had fatal ADRs; of these, six patients had fatal ADRs classified as 'drugrelated', which were: interstitial lung disease $(n=3)$, pneumonia $(n=2)$, respiratory failure $(n=2)$ and heart failure $(n=1)$; three patients had fatal ADRs which were deemed as 'unassessable' by the investigating physician: gingival cancer $(n=1)$, disseminated intravascular coagulation $(n=1)$ and Epstein-Barr virus infection $(n=1)$; we have deemed one fatal ADR (HL, $n=1$ ) as 'unassessable' as we were unable to obtain the physician's judgment.

With regards to the assessment of ADRSIs, peripheral neuropathy events were reported in $39.4 \%$ of overall patients, with $6.7 \%$ of patients experiencing grade $\geq 3$ peripheral neuropathy (Table 5). Of 17 patients who had grade 2 peripheral neuropathy prior to starting brentuximab vedotin, two (11.8\%) patients reported worsening to grade 3 . The only patient having grade 3 peripheral neuropathy prior to starting brentuximab vedotin also reported grade 3 peripheral neuropathy during treatment. Of 35 events of grade $\geq 3$ peripheral neuropathy that occurred during brentuximab vedotin treatment, five (14.3\%) required treatment interruptions and $23(65.7 \%)$ resulted in treatment discontinuation (Supplementary Table 2).

The incidence of infections in the safety population was $13.0 \%$ (Table 5). Concomitant prophylaxis was given to 184 (64.8\%) patients and included sulfamethoxazole/trimethoprim (ST; 53.5\%), antifungal agents other than ST (26.1\%), antiviral agents (19.7\%), and antibiotics and synthetic antibacterial drugs (14.1\%). The incidence of infections was higher in patients who received prophylactic treatment $(15.8 \%)$ than in those who did not $(8.0 \%)$ and in patients who underwent HSCT prior to study initiation than in those who did not (Supplementary Table 3).

In the overall population, the incidence of grade $\geq 3$ neutropenia was $22.5 \%$ (Table 5), which included $3.2 \%$ of patients with febrile neutropenia. Of 117 events of grade $\geq 3$ neutropenia, 28 (23.9\%) required dose-adjustment and two $(1.7 \%)$ resulted in treatment discontinuation (Supplementary Table 4). 
Table 2 Exposure to brentuximab vedotin

\begin{tabular}{llll}
\hline & $\begin{array}{l}\text { Total } \\
(N=284)^{\mathrm{a}}\end{array}$ & $\begin{array}{l}\text { HL } \\
(n=182)\end{array}$ & $\begin{array}{l}\text { sALCL } \\
(n=101)\end{array}$ \\
\hline Initial dose, mg/kg & & & \\
$<1.2$ & $7(2.5)$ & $4(2.2)$ & $3(3.0)$ \\
1.2 & $17(6.0)$ & $9(4.9)$ & $8(7.9)$ \\
$>1.2-<1.8$ & $12(4.2)$ & $9(4.9)$ & $3(3.0)$ \\
1.8 & $247(87.0)$ & $159(87.4)$ & $87(86.1)$ \\
$>1.8$ & $1(0.4)$ & $1(0.5)$ & 0 \\
Mean dose per cycle is 1.8 mg/kg & $214(75.4)$ & $141(77.5)$ & $72(71.3)$ \\
Median number of cycles received (range) & $5.0(1-17)$ & $5.5(1-16)$ & $4.0(1-17)$ \\
Reasons for study drug discontinuation (cumulative) $)$ & & $25(24.8)$ \\
Treatment objective was achieved & $60(21.1)$ & $35(19.2)$ & $21(20.8)$ \\
AE & $63(22.2)$ & $42(23.1)$ & 0 \\
Discontinuation of clinic visits & $1(0.4)$ & $1(0.5)$ & $4(4.0)$ \\
Transfer to another clinic & $9(3.2)$ & $5(2.7)$ & $40(39.6)$ \\
Lack of efficacy & $115(40.5)$ & $74(40.7)$ & $18(17.8)$ \\
Others & $53(18.7)$ & $34(18.7)$ & $20(19.8)$ \\
Received HSCT after BV treatment & $56(19.7)$ & $36(19.8)$ & $9(45.0)$ \\
Autologous & $35(62.5)$ & $26(72.2)$ & $11(55.0)$ \\
Allogenic & $21(37.5)$ & $10(27.8)$ & $13(12.9)$ \\
Received other combination therapies & $31(10.9)$ & $18(9.9)$ & \\
\hline
\end{tabular}

${ }^{\mathrm{a}}$ Includes one patient with diffuse large B-cell lymphoma

Number of cases (\%) are shown unless indicated otherwise

$A E$ adverse event, $B V$ brentuximab vedotin, $H L$ Hodgkin lymphoma, HSCT hematopoietic stem cell transplant, $s A L C L$ systemic anaplastic large cell lymphoma
The incidence of infusion reactions in the overall population was $6.7 \%$ (Table 5). Common infusion reactions included fever $(4.2 \%)$, erythema $(1.1 \%)$, chills $(0.7 \%)$, pruritus $(0.7 \%)$, and hot flashes $(0.7 \%)$. Of the 1099 brentuximab vedotin injections for which premedication was administered, infusion reactions occurred in $13(1.2 \%)$ instances. Of the 788 injections without premedication, infusion reactions occurred in six $(0.8 \%)$ instances.

The incidence of pulmonary toxicity was $4.6 \%$ (grade $\geq 3,3.9 \%$ ) in the overall population (Table 5), including interstitial lung disease in $3.9 \%$ of patients, respiratory failure in $0.7 \%$, and acute respiratory distress syndrome in $0.4 \%$. Among 255 patients who did not have a history of pulmonary disorders prior to brentuximab vedotin, grade 3,4 , or 5 pulmonary toxicity occurred in four $(1.6 \%)$, two $(0.8 \%)$, and two $(0.8 \%)$ patients, respectively. Among 29 patients with a history of or ongoing pulmonary disorders, each of grade 1,2 , or 3 pulmonary toxicity occurred in one patient (3.4\%), and grade 5 pulmonary toxicity occurred in two $(6.9 \%)$ patients; all five patients had prior or ongoing interstitial lung disease.

\section{Effectiveness}

Responses in patients with HL and sALCL per imaging assessment methodology, utilizing computed tomography (CT) only (without PET subgroup) or PET (with PET subgroup), are shown in Fig. 2. Patients for whom imaging assessment data were not available were excluded. In 162 evaluable patients with HL, the overall response rate (ORR), including complete response (CR), unconfirmed $\mathrm{CR}(\mathrm{CRu})$, and partial response (PR), was 50.0\%, and the $\mathrm{CR} / \mathrm{CRu}$ rate was $30.9 \%$. In 79 evaluable patients with sALCL, the ORR was $62.0 \%$ and the $\mathrm{CR} / \mathrm{CRu}$ rate was $36.7 \%$.

The analysis population for OS included 179 patients with HL and 101 patients with sALCL. Kaplan-Meier analyses of OS for patients with HL and sALCL are shown in Supplementary Fig. 1. For OS assessments, patients with HL and sALCL were followed up for a median of 284 days (range 4-844) and 218 days (range 3-799), achieving 1-year survival rates of $82.7 \%$ (95\% confidence interval [CI] 75.4, 88.0) and $79.3 \%$ (95\% CI 68.4, 86.7), respectively. Of the 51 patients with HL or sALCL who died, 34 patients died from 
Table 3 Common ADRs occurring in $\geq 2 \%$ of all patients

\begin{tabular}{|c|c|c|c|c|c|c|}
\hline \multirow{2}{*}{$\begin{array}{l}\text { ADRs, } n(\%) \\
\text { System organ class } \\
\text { Preferred term }\end{array}$} & \multicolumn{3}{|l|}{ Grade $\geq 3$} & \multicolumn{3}{|l|}{ Any Grade } \\
\hline & $\begin{array}{l}\text { Total }^{\mathrm{a}} \\
(N=284)\end{array}$ & $\begin{array}{l}\mathrm{HL} \\
(n=182)\end{array}$ & $\begin{array}{l}\text { sALCL } \\
(n=101)\end{array}$ & $\begin{array}{l}\text { Total }^{\mathrm{a}} \\
(N=284)\end{array}$ & $\begin{array}{l}\text { HL } \\
(n=182)\end{array}$ & $\begin{array}{l}\text { sALCL } \\
(n=101)\end{array}$ \\
\hline Overall ADRs & NA & NA & NA & $211(74.3)$ & $139(76.4)$ & $71(70.3)$ \\
\hline Infections and infestations & $19(6.7)$ & $11(6.0)$ & $8(7.9)$ & $37(13.0)$ & $24(13.2)$ & $13(12.9)$ \\
\hline Pneumonia & $6(2.1)$ & $3(1.7)$ & $3(3.0)$ & $7(2.5)$ & $3(1.7)$ & $4(4.0)$ \\
\hline Blood and lymphatic system disorders & $79(27.8)$ & $53(29.1)$ & $25(24.8)$ & 119 (41.9) & $78(42.9)$ & $40(39.6)$ \\
\hline Anemia & $3(1.1)$ & $3(1.7)$ & 0 & $6(2.1)$ & $6(3.3)$ & 0 \\
\hline Febrile neutropenia & $7(2.5)$ & $4(2.2)$ & $3(3.0)$ & $9(3.2)$ & $4(2.2)$ & $5(5.0)$ \\
\hline Leukopenia & $7(2.5)$ & $6(3.3)$ & $1(1.0)$ & $10(3.5)$ & $8(4.4)$ & $2(2.0)$ \\
\hline Lymphopenia & $15(5.3)$ & $12(6.6)$ & $3(3.0)$ & $20(7.0)$ & $15(8.2)$ & $5(5.0)$ \\
\hline Neutropenia & $63(22.2)$ & $41(22.5)$ & $21(20.8)$ & $98(34.5)$ & $64(35.2)$ & $33(32.7)$ \\
\hline Thrombocytopenia & $8(2.8)$ & $8(4.4)$ & 0 & $16(5.6)$ & $15(8.2)$ & $1(1.0)$ \\
\hline Metabolism and nutrition disorders & $5(1.8)$ & $4(2.2)$ & $1(1.0)$ & $10(3.5)$ & $6(3.3)$ & $4(4.0)$ \\
\hline Nervous system disorders & $20(7.0)$ & $17(9.3)$ & $3(3.0)$ & $114(40.1)$ & $76(41.8)$ & $38(37.6)$ \\
\hline Peripheral motor neuropathy & $8(2.8)$ & $7(3.9)$ & $1(1.0)$ & $15(5.3)$ & $9(5.0)$ & $6(5.9)$ \\
\hline Peripheral sensory neuropathy & $18(6.3)$ & $15(8.2)$ & $3(3.0)$ & $111(39.1)$ & $74(40.7)$ & $37(36.6)$ \\
\hline Respiratory, thoracic and mediastinal disorders & $11(3.9)$ & $8(4.4)$ & $3(3.0)$ & $20(7.0)$ & $17(9.3)$ & $3(3.0)$ \\
\hline Interstitial lung disease & $9(3.2)$ & $6(3.3)$ & $3(3.0)$ & $11(3.9)$ & $8(4.4)$ & $3(3.0)$ \\
\hline Gastrointestinal disorder & $4(1.4)$ & $2(1.1)$ & $2(2.0)$ & $19(6.7)$ & $13(7.1)$ & $6(5.9)$ \\
\hline Diarrhea & $1(0.4)$ & 0 & $1(1.0)$ & $6(2.1)$ & $4(2.2)$ & $2(2.0)$ \\
\hline Nausea & 0 & 0 & 0 & $6(2.1)$ & $4(2.2)$ & $2(2.0)$ \\
\hline Skin and subcutaneous tissue disorders & $8(2.8)$ & $6(3.3)$ & $2(2.0)$ & $37(13.0)$ & $27(14.8)$ & $10(9.9)$ \\
\hline Rashes & $3(1.1)$ & $3(1.7)$ & 0 & $12(4.2)$ & $11(6.0)$ & $1(1.0)$ \\
\hline General disorders and administration site conditions & $3(1.1)$ & $1(0.6)$ & $2(2.0)$ & $15(5.3)$ & $8(4.4)$ & $7(6.9)$ \\
\hline Fever & $2(0.7)$ & $1(0.6)$ & $1(1.0)$ & $9(3.2)$ & $6(3.3)$ & $3(3.0)$ \\
\hline Investigations $^{b}$ & $3(1.1)$ & $2(1.1)$ & $1(1.0)$ & $24(8.5)$ & $12(6.6)$ & $12(11.9)$ \\
\hline Alanine aminotransferase increased & $1(0.4)$ & $1(0.6)$ & 0 & $9(3.2)$ & $8(4.4)$ & $1(1.0)$ \\
\hline Aspartate aminotransferase increased & $1(0.4)$ & $1(0.6)$ & 0 & $10(3.5)$ & $8(4.4)$ & $2(2.0)$ \\
\hline Injury, poisoning and procedural complications & $2(0.7)$ & $1(0.6)$ & $1(1.0)$ & $20(7.0)$ & $12(6.6)$ & $8(7.9)$ \\
\hline Infusion reactions & $1(0.4)$ & 0 & $1(1.0)$ & $19(6.7)$ & $11(6.0)$ & $8(7.9)$ \\
\hline
\end{tabular}

${ }^{a}$ Includes one patient with diffuse large B-cell lymphoma

${ }^{\mathrm{b}}$ Two patients with sALCL had grade unknown

$A D R$ adverse drug reaction, $H L$ Hodgkin lymphoma, $N A$ not available, $s A L C L$ systemic anaplastic large cell lymphoma

progression of the primary disease and 17 patients from AEs excluding the primary disease.

\section{Discussion}

The aim of this study was to collect safety information on brentuximab vedotin in the clinical setting following Japanese regulatory approval for use in patients with relapsed or refractory HL and sALCL. In contrast to clinical studies, this study enrolled patients with a wider age range, level of functioning and treatment history, as expected in a postmarketing surveillance study. Our study enrolled a higher proportion of elderly patients compared with the pivotal clinical studies: the median age of patients with HL and
sALCL was 60.5 and 64.0 years, respectively; in the pivotal phase II studies, they were 31 and 52 years, respectively $[3,4]$. The patients' ECOG performance status at baseline was $\geq 2$ in $19.8 \%$ of patients with HL and $28.7 \%$ of those with SALCL in this study, whereas the pivotal clinical studies included only patients with an ECOG performance status of 0 or $1[3,4]$. We have calculated the occurrence of ADRs by patient background characteristics and found no specific factors (data not shown). Thus, compared with the previous clinical studies, our study shows an acceptable safety profile of brentuximab vedotin in a wider patient population.

HL and SALCL patients received a median of 5.5 and 4 cycles of treatment, respectively, which were shorter than the respective medians of 9 and 7 cycles in the pivotal phase 2 studies [3, 4]. Lack of efficacy accounted for $40.5 \%$ of 
Table 4 Serious ADRs with incidence of $\geq 1 \%$ in the total population

\begin{tabular}{llll}
\hline $\begin{array}{l}\text { Serious ADRs, } n(\%) \\
\text { System organ class } \\
\text { Preferred term }\end{array}$ & $\begin{array}{l}\text { Total }^{\mathrm{a}} \\
(N=284)\end{array}$ & $\begin{array}{l}\text { HL } \\
(n=182)\end{array}$ & $\begin{array}{l}\text { sALCL } \\
(n=101)\end{array}$ \\
\hline Infections and infestations & $19(6.7)$ & $12(6.6)$ & $7(6.9)$ \\
Pneumonia & $6(2.1)$ & $3(1.7)$ & $3(3.0)$ \\
Sepsis & $3(1.1)$ & $1(0.6)$ & $2(2.0)$ \\
Pneumocystis jirovecii pneumonia & $3(1.1)$ & $2(1.1)$ & $1(1.0)$ \\
Blood and lymphatic system disorders & $20(7.0)$ & $14(7.7)$ & $6(5.9)$ \\
Febrile neutropenia & $4(1.4)$ & $2(1.1)$ & $2(2.0)$ \\
Neutropenia & $14(4.9)$ & $12(6.6)$ & $2(2.0)$ \\
Nervous system disorders & $7(2.5)$ & $6(3.3)$ & $1(1.0)$ \\
Peripheral motor neuropathy & $5(1.8)$ & $4(2.2)$ & $1(1.0)$ \\
Peripheral sensory neuropathy & $6(2.1)$ & $5(2.8)$ & $1(1.0)$ \\
Respiratory, thoracic and mediastinal disorders & $13(4.6)$ & $10(5.5)$ & $3(3.0)$ \\
Interstitial lung disease & $11(3.9)$ & $8(4.4)$ & $3(3.0)$ \\
\hline
\end{tabular}

${ }^{\mathrm{a}}$ Includes one patient with diffuse large B-cell lymphoma

$A D R$ adverse drug reaction, $H L$ Hodgkin lymphoma, $s A L C L$ systemic anaplastic large cell lymphoma

\begin{tabular}{|c|c|c|c|c|c|c|}
\hline \multirow[t]{2}{*}{ ADRSI $n(\%)$} & \multicolumn{3}{|l|}{ Grade $\geq 3$} & \multicolumn{3}{|l|}{ Any grade } \\
\hline & $\begin{array}{l}\text { Total }^{\mathrm{a}} \\
(N=284)\end{array}$ & $\begin{array}{l}\text { HL } \\
(n=182)\end{array}$ & $\begin{array}{l}\text { sALCL } \\
(n=101)\end{array}$ & $\begin{array}{l}\text { Total }^{\mathrm{a}} \\
(N=284)\end{array}$ & $\begin{array}{l}\mathrm{HL} \\
(n=182)\end{array}$ & $\begin{array}{l}\text { sALCL } \\
(n=101)\end{array}$ \\
\hline Peripheral neuropathy ${ }^{b}$ & $19(6.7)$ & $16(8.8)$ & $3(3.0)$ & $112(39.4)$ & $74(40.7)$ & $38(37.6)$ \\
\hline Infections $^{c}$ & $19(6.7)$ & $11(6.0)$ & $8(7.9)$ & $37(13.0)$ & $24(13.2)$ & $13(12.9)$ \\
\hline Neutropenia $^{\mathrm{d}}$ & $64(22.5)$ & $42(23.1)$ & $21(20.8)$ & $100(35.2)$ & $65(35.7)$ & $34(33.7)$ \\
\hline Infusion reactions $^{\mathrm{e}}$ & $1(0.4)$ & 0 & $1(1.0)$ & $19(6.7)$ & $11(6.0)$ & $8(7.9)$ \\
\hline Pulmonary toxicities ${ }^{\mathrm{f}}$ & $11(3.9)$ & $8(4.4)$ & $3(3.0)$ & $13(4.6)$ & $10(5.5)$ & $3(3.0)$ \\
\hline
\end{tabular}

${ }^{a}$ Includes one patient with diffuse large B-cell lymphoma

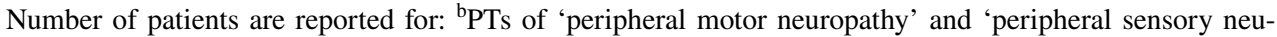
ropathy'; 'SOC of 'infections and infestations'; 'PTs of 'neutropenia' and 'febrile neutropenia'; 'PT of 'infusion reaction'; ${ }^{\mathrm{f}} \mathrm{PTs}$ of 'interstitial lung disease', 'respiratory failure' and 'acute respiratory distress syndrome'

$A D R S I$ adverse drug reaction of special interest, $H L$ Hodgkin lymphoma, $P T$ Preferred term, $s A L C L$ systemic anaplastic large cell lymphoma, SOC system organ class treatment discontinuations, followed by occurrence of AEs (22.2\%), highlighting the importance of management of AEs.

Of the ten patients who had fatal ADRs, six patients had ADRs deemed as related to the drug. The causality between the other four fatal ADRs and the drug were deemed as unassessable. The unassessable fatal ADRs included gingival cancer, which was reported approximately 3 months after the patient had received the last dose of brentuximab vedotin for treatment of sALCL. As the patient had received multiple therapies other than brentuximab vedotin, including other anti-cancer drugs and radiotherapy, causality between brentuximab vedotin and this secondary malignancy could not be established in this study.

The incidence of infections in patients who received prophylactic treatment was higher than in those who did not. It is plausible that the patients who were prescribed prophylaxis had reasons for the investigating physician to be concerned about infection, and thus may have been more susceptible to infection. It is thus difficult to derive a conclusion from the results.

The incidence of peripheral neuropathy of any grade in the total population was $39.4 \%$. No correlations were found between the occurrence of peripheral neuropathy and patient clinical factors or the amount of initial, mean, or the cumulative (at week 3) doses of brentuximab vedotin (data not shown). The time to occurrence of peripheral neuropathy from treatment initiation was within 9 weeks in $61 \%$ of the patients (94/153), within 27 weeks in 90\% (138/153), and within 37 weeks in $98 \%$ (150/153). Grade $\geq 3$ peripheral neuropathy was reported in 35 events, leading to 23 discontinuation of brentuximab vedotin. In contrast, only four 
HL

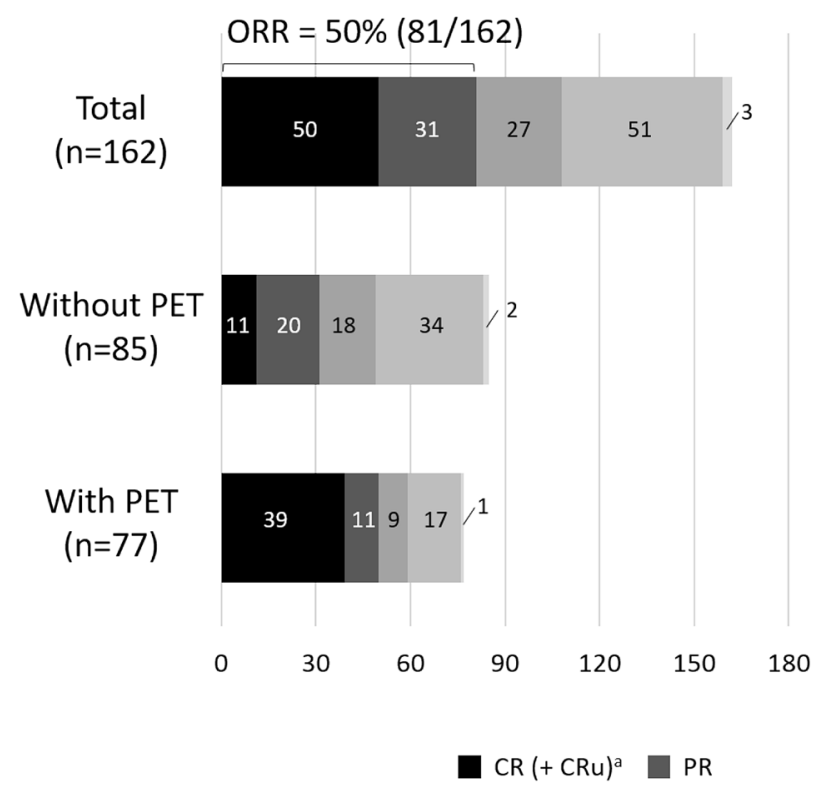

Fig. 2 Antitumor effect of brentuximab vedotin. ${ }^{\mathrm{a}} \mathrm{A}$ response of $\mathrm{CRu}$ was only used in study sites where PET was not utilized. $C R$ complete response, $C R u$ unconfirmed $\mathrm{CR}, H L$ Hodgkin lymphoma, ORR

events with grade 2 peripheral neuropathy $(n=54)$ and no grade 1 events $(n=64)$, resulted in discontinuation. These data suggest that brentuximab vedotin dose modifications should be introduced when patients experience grade 2 peripheral neuropathy, prior to worsening to grade $\geq 3$.

The overall incidence of grade $\geq 3$ neutropenia in our study was $22.5 \%$, which appears similar to the rates of $19 \%$ and $21 \%$ reported in the pivotal phase 2 studies in HL [3] and sALCL [4], respectively. Of the 117 grade $\geq 3$ events, 28 required dose-adjustment and only two events resulted in treatment discontinuation, suggesting that utilization of the dose-modification guidelines and granulocyte colonystimulating factor in clinical practice provided effective management of neutropenia.

Infusion reactions are frequently associated with the use of monoclonal antibodies, and premedication is generally recommended. In the present study, $65.1 \%$ of patients received premedication based on their physician's decision. The rate of infusion reactions was $0.8 \%$ of injections without premedication and $1.2 \%$ of injections with premedication, which does not support routine use of premedication before administration of brentuximab vedotin in Japan.

No correlations were found between the occurrence of pulmonary toxicity and prior radiotherapy or smoking history (data no shown); however, the risk of pulmonary toxicity with brentuximab vedotin was elevated in patients with prior or ongoing pulmonary disorders. Of
sALCL

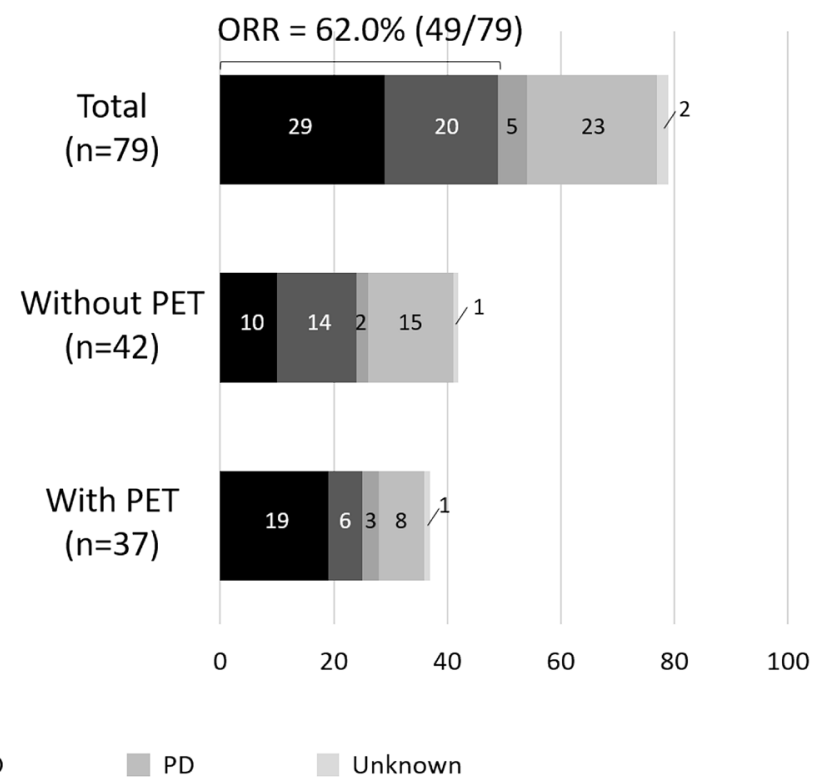

overall response rate, $P D$ progressive disease, $P E T$ positron emission tomography, $P R$ partial response, $s A L C L$ systemic anaplastic large cell lymphoma, $S D$ stable disease

29 such patients enrolled in the present study, five (17.2\%) experienced pulmonary toxicity as a result of brentuximab vedotin treatment. In contrast, there were only eight cases $(3.1 \%)$ of pulmonary toxicity among the 255 patients without a history of pulmonary disorders. As such, careful monitoring is needed when using brentuximab vedotin in patients with either prior or ongoing pulmonary disorders.

The current study demonstrated the effectiveness of brentuximab vedotin in patients with HL or sALCL in the clinical practice setting. Among evaluable patients for whom imaging data were available, ORR assessed by CT and PET were $36.5 \%$ (31/85) and 64.9\% (50/77), respectively, in the HL population; and were $57.1 \%(24 / 42)$ and $67.6 \%$ (25/37), respectively, in sALCL. The response rate in this study appears to be somewhat lower than those reported in the pivotal phase 2 clinical studies, which demonstrated an ORR of $75 \%$ and $86 \%$ in HL and sALCL, respectively [3, 4]. Care is needed when interpreting the effectiveness result, which was an exploratory endpoint of this observational study; response assessment to treatment was conducted as a part of routine medical care including laboratory tests, physical examinations or standard CT scans. ORR assessment was not mandatory; thus, the implementation rate of PET scans was low (47.3\% [114/241]). In addition, the median follow-up period for the OS assessment was less than a year for both HL (284 days) and sALCL (218 days). 
The aim of this surveillance study was to collect safety data of brentuximab vedotin in clinical practice in Japan, including data on the occurrence and severity of ADRs. The study provides limited information on associations between the ADRs and other factors such as concomitant treatments and comorbidities.

In conclusion, in comparison to the toxicity profile observed in clinical studies in the US and Japan, no additional safety signals were observed in this post-marketing surveillance study of 284 patients treated with brentuximab vedotin in clinical practice between April and September 2014 in Japan. However, as rare, but potentially fatal, ADRs such as pulmonary toxicity have been consistently observed across studies, close monitoring may be warranted especially in patients with either prior or ongoing pulmonary disorders.

Acknowledgements This study was funded by Takeda Pharmaceutical Company Limited. The authors would like to thank all patients and their families, and all investigators for their valuable contributions to this study. The authors are grateful to Yoshihide Kakimoto and Tomoko Yanai of Takeda Pharmaceutical Co. Ltd. for reviewing and providing valuable advice on this manuscript. The authors acknowledge Fumiko Shimizu, of Ashfield Healthcare Communications K.K., part of UDG Healthcare plc, for medical writing support during the development of this manuscript, which was funded Takeda Pharmaceutical Co. Ltd. The corresponding author had full access to all the study data and had final responsibility for the decision to submit for publication.

\section{Compliance with ethical standards}

Conflict of interest KI reports non-financial support from Takeda during the conduct of the study; grants, personal fees or both from Takeda, Chugai, Celgene, Novartis, Abbvie, Janssen, Kyowa Kirin, Eisai, Merck, Sharp \& Dohme, Astra Zeneca, FUJIFILM Toyama Chemical, Ono Pharmaceutical, Nihon Mediphysics, Dainihon Sumitomo, Bayer, HUYA Japan, Bristol Myers Squibb, Mundi Pharma, Otsuka, Daiichi Sankyo, Astellas and Asahi Kasei Pharma, outside the submitted work. MO reports personal fees from Celgene, Denovo Biopharma, Mundi Pharma, Meiji Seika Pharma, Celtrion, Verastem, Eisai and Teva Takeda, outside the submitted work. KT reports grants and personal fees from Takeda during the conduct of the study; grants, personal fees or both from Merck, Celgene, Daiichi Sankyo, HUYA Bioscience International, Mundipharma, Ono Pharmaceutical, Zenyaku Kogyo, Bristol Myers Squibb, Chugai, Eisai, Janssen, Kyowa Kirin, Meiji Seika, Solasia Pharma, Verastem, Yakult and Abbvie, outside the submitted work.
KH report personal fees from Takeda, outside the submitted work. SS, $\mathrm{MN}$ and $\mathrm{MH}$ report employment with Takeda Pharmaceutical Co. Ltd. during the conduct of the study.

\section{References}

1. van de Donk NW, Dhimolea E. Brentuximab vedotin. MAbs. 2012;4(4):458-65.

2. Seattle Genetics Inc. ADCETRIS ${ }^{\circledR}$ (brentuximab vedotin) for injection, for intravenous use, US Prescribing Information. 2019. https://seagendocs.com/Adcetris_Full_Ltr_Master.pdf.

3. Younes A, Gopal AK, Smith SE, Ansell SM, Rosenblatt JD, Savage KJ, et al. Results of a pivotal phase II study of brentuximab vedotin for patients with relapsed or refractory Hodgkin's lymphoma. J Clin Oncol. 2012;30(18):2183-9.

4. Pro B, Advani R, Brice P, Bartlett NL, Rosenblatt JD, Illidge T, et al. Brentuximab vedotin (SGN-35) in patients with relapsed or refractory systemic anaplastic large-cell lymphoma: results of a phase II study. J Clin Oncol. 2012;30(18):2190-6.

5. Chen R, Gopal AK, Smith SE, Ansell SM, Rosenblatt JD, Savage KJ, et al. Five-year survival and durability results of brentuximab vedotin in patients with relapsed or refractory Hodgkin lymphoma. Blood. 2016;128(12):1562-6.

6. Pro B, Advani R, Brice P, Bartlett NL, Rosenblatt JD, Illidge T, et al. Five-year results of brentuximab vedotin in patients with relapsed or refractory systemic anaplastic large cell lymphoma. Blood. 2017;130(25):2709-17.

7. Sabet Y, Ramirez S, Rosell Cespedes E, Rensoli Velasquez M, Porres-Munoz M, Gaur S, et al. Severe acute pulmonary toxicity associated with brentuximab in a patient with refractory Hodgkin's lymphoma. Case Rep Pulmonol. 2016;2016:2359437.

8. Ogura M, Tobinai K, Hatake K, Ishizawa K, Uike N, Uchida $\mathrm{T}$, et al. Phase I/II study of brentuximab vedotin in Japanese patients with relapsed or refractory CD30-positive Hodgkin's lymphoma or systemic anaplastic large-cell lymphoma. Cancer Sci. 2014;105(7):840-6.

9. Takeda Pharmaceutical Co. Ltd. ADCETRIS ${ }^{\circledR}$, Japan package insert [in Japanese]. 2018. https://www.info.pmda.go.jp/go/ pack/4291425D1021_1_11/.

10. Cheson BD, Pfistner B, Juweid ME, Gascoyne RD, Specht L, Horning SJ, et al. Revised response criteria for malignant lymphoma. J Clin Oncol. 2007;25(5):579-86.

Publisher's Note Springer Nature remains neutral with regard to jurisdictional claims in published maps and institutional affiliations. 